\title{
Multiple Positive Solutions for a System of Caputo Fractional $p$-Laplacian Boundary Value Problems
}

\author{
Zhi-Wei Lv $\mathbb{D}^{1},{ }^{1}$ Jie Liu $(\mathbb{D})^{2}$ and Jiafa Xu ${ }^{3}{ }^{3}$ \\ ${ }^{1}$ Department of Mathematics and Physics, Anyang Institute of Technology, Anyang, Henan 455000, China \\ ${ }^{2}$ School of Mathematics and Information Science, Henan Polytechnic University, Jiaozuo, Henan 454000, China
}

${ }^{3}$ School of Mathematical Sciences, Qufu Normal University, Qufu, Shandong 273165, China

Correspondence should be addressed to Jie Liu; jzyzliujie@hpu.edu.cn

Received 1 March 2020; Accepted 13 May 2020; Published 2 June 2020

Academic Editor: Cornelio Posadas-Castillo

Copyright (C) 2020 Zhi-Wei Lv et al. This is an open access article distributed under the Creative Commons Attribution License, which permits unrestricted use, distribution, and reproduction in any medium, provided the original work is properly cited.

In this work, we are pleased to investigate multiple positive solutions for a system of Caputo fractional $p$-Laplacian boundary value problems, and we also provide an example for illustrating our main results.

\section{Introduction}

In this work, we are pleased to discuss the positive solutions for the following system of Caputo fractional $p$-Laplacian boundary value problems:

$$
\begin{cases}{ }^{c} D_{t}^{\nu_{1}}\left(\varphi_{p}\left({ }^{c} D_{t}^{\mu_{1}} u(t)\right)\right)+g_{1}(t, u(t), v(t))=0, & 0<t<1, \\ { }^{c} D_{t}^{\nu_{2}}\left(\varphi_{p}\left({ }^{c} D_{t}^{\mu_{2}} v(t)\right)\right)+g_{2}(t, u(t), v(t))=0, & 0<t<1, \\ \alpha_{1} u(0)-\beta_{1} u(1)=0, \gamma_{1} u^{\prime}(0)-\delta_{1} u^{\prime}(1)=0, & { }^{c} D_{t}^{\mu_{1}} u(0)=0,{ }^{c} D_{t}^{\mu_{1}} u(1)=b_{1}{ }^{c} D_{t}^{\mu_{1}} u\left(\xi_{1}\right), \\ \alpha_{2} v(0)-\beta_{2} v(1)=0, \gamma_{2} v^{\prime}(0)-\delta_{2} v^{\prime}(1)=0, & { }^{c} D_{t}^{\mu_{2}} v(0)=0,{ }^{c} D_{t}^{\mu_{2}} v(1)=b_{2}{ }^{c} D_{t}^{\mu_{2}} v\left(\xi_{2}\right),\end{cases}
$$

where ${ }^{c} D_{t}^{\mu_{i}},{ }^{c} D_{t}^{\nu_{i}}(i=1,2)$ are the fractional derivatives of Caputo sense with $\mu_{i}, v_{i} \in(1,2) ; \varphi_{p}$ is the $p$-Laplacian, i.e., $\varphi_{p}(x)=|x|^{p-2} x$ with $p>1, x \in \mathbb{R} ;$ and the constants $\alpha_{i}, \beta_{i}, \gamma_{i}, \delta_{i}, b_{i}, \xi_{i}(i=1,2)$ and the functions $g_{i}(i=1,2)$ satisfy the following conditions:

(H0) $\quad \alpha_{i}>\beta_{i}>0, \quad \gamma_{i}>\delta_{i}>0, \quad b_{i} \geq 0, \xi_{i} \in(0,1) \quad$ with $b_{i}^{p-1} \xi_{i} \in[0,1)$

(H1) $g_{i}(i=1,2)$ are nonnegative continuous functions on $[0,1] \times \mathbb{R}^{+} \times \mathbb{R}^{+}$, where $\mathbb{R}^{+}:=[0,+\infty)$
As a generalization of integer-order equations, fractional-order equations can effectively describe various materials and physical processes with memory and genetic properties. It has a large number of applications in our society, such as biology, chemical kinetics, electromagnetics, transmission and diffusion, and automatic control. Recently, there many researchers pay their attentions to studying the existence of solutions for various types of fractional-order equations by use of fixed point theorems, upper and lower solution methods, and monotone iterative techniques. For 
instance, we refer the readers to [1-25] and references therein.

In [1], by using the method of upper and lower solutions and the Schauder fixed point theorem, Vong investigated the positive solutions for the following nonlocal fractional boundary value problem:

$$
\left\{\begin{array}{l}
{ }^{c} D_{t}^{\alpha} u(t)+f(t, u(t))=0, \quad 0<t<1, \\
u^{\prime}(0)=\cdots=u^{(n-1)}(0)=0, u(1)=\int_{0}^{1} u(s) \mathrm{d} \mu(s),
\end{array}\right.
$$

where $n \geq 2, \alpha \in(n-1, n)$, and $\mu(s)$ is a function of bounded variation. $f$ may be singular at $t=1$.

In [2], Wang and Yang studied the integral boundary value problem of Caputo sense:

$$
\left\{\begin{array}{l}
{ }^{c} D_{t}^{q} \chi(t)=f(t, \chi(t)), \quad 0<t<1, \\
\alpha \chi(0)-\beta \chi(1)=\int_{0}^{1} h(t) \chi(t) \mathrm{d} t, \gamma \chi^{\prime}(0)-\delta \chi^{\prime}(1)=\int_{0}^{1} g(t) \chi(t) \mathrm{d} t .
\end{array}\right.
$$

By the Leggett-Williams fixed point theorem, they obtained multiple positive solutions when the nonlinearity $f$ is bounded from below. Moreover, when $f$ is asymptotically linear at infinity, they also obtained an existence theorem.

In [3], Wang et al. adopted the theory of mixed monotone operators to obtain a unique positive solution for the mixed fractional boundary value problems involving the $p$-Laplacian:

$$
\left\{\begin{array}{l}
{ }^{c} D_{t}^{\alpha} \varphi_{p}\left(D_{0+}^{\beta} v(t)\right)+\lambda f\left(t, v(t), D_{0+}^{\beta_{1}} v(t), \ldots, D_{0+}^{\beta_{n-2}} v(t)\right)=0, \quad 0<t<1, \\
v(0)=D_{0+}^{v_{i}} v(0)=D_{0+}^{\beta} v(0)=\left(\varphi_{p}\left(D_{0+}^{\beta} v(0)\right)\right)^{\prime}=0, \quad i=1, \ldots, n-2, \\
D_{0+}^{r_{0}} v(1)=\lambda_{1} \int_{0}^{1} h_{1}(s) D_{0+}^{r_{1}} v(s) \mathrm{d} A_{1}(s)+\lambda_{2} \int_{0}^{\eta} h_{2}(s) D_{0+}^{r_{2}} v(s) \mathrm{d} A_{2}(s)+\lambda_{3} \sum_{j=1}^{\infty} \rho_{j} D_{0+}^{r_{3}} v\left(\eta_{j}\right),
\end{array}\right.
$$

where ${ }^{c} D_{t}^{\alpha}$ is the Caputo fractional derivative and $D_{0+}^{\beta}, D_{0+}^{\beta_{i}}, D_{0^{\prime}}^{\nu_{i}}$, and $D_{0_{+}}^{r_{i}}$ are the Riemann-Liouville fractional derivatives.

Recently, coupled systems of fractional differential equations have also been investigated by many authors.
Some results on the direction can be found in a series of papers [11-25] and the references cited therein.

In [11], Wang utilized the Guo-Krasnosel'skii fixed point theorem to investigate the multiple positive solutions for the mixed fractional $p$-Laplacian differential system:

$$
\begin{cases}D_{0+}^{\beta_{1}}\left(\varphi_{p_{1}}\left({ }^{c} D_{t}^{\alpha_{1}} u t(t)\right)\right)+f_{1}(t, u(t), v(t))=0, & 0<t<1, \\ D_{0+}^{\beta_{2}}\left(\varphi_{p_{2}}\left({ }^{c} D_{t}^{\alpha_{2}} v t(t)\right)\right)+f_{2}(t, u(t), v(t))=0, & 0<t<1, \\ u^{\prime}(0)=u^{\prime \prime}(0)=\cdots=u^{(n-1)}(0)=0, & u(1)=\mu_{1} \int_{0}^{1} a(s) v(s) \mathrm{d} A_{1}(s), \\ v^{\prime}(0)=v^{\prime \prime}(0)=\cdots=v^{(m-1)}(0)=0, & v(1)=\mu_{2} \int_{0}^{1} b(s) u(s) \mathrm{d} A_{2}(s), \\ { }^{c} D_{t}^{\alpha_{1}} u(0)=0, & { }^{c} D_{t}^{\alpha_{1}} u(1)=\varepsilon_{1}{ }^{c} D_{t}^{\alpha_{1}} u\left(\eta_{1}\right), \\ { }^{c} D_{t}^{\alpha_{2}} v(0)=0, & { }^{c} D_{t}^{\alpha_{2}} v(1)=\varepsilon_{2}{ }^{c} D_{t}^{\alpha_{2}} v\left(\eta_{2}\right),\end{cases}
$$

where $D_{0+}^{\beta_{i}}$ are the Riemann-Liouville fractional derivatives, ${ }^{c} D_{t}^{\alpha_{i}}$ the Caputo fractional derivatives, and $\int_{0}^{1} a(s) v(s) \mathrm{d} A_{1}(s)$ and $\int_{0}^{1} b(s) u(s) \mathrm{d} A_{2}(s)$ are the Riemann-Stieltjes integrals.

In [12], Rao studied the system of fractional $p$-Laplacian differential equations:

$$
\begin{cases}D_{0+}^{\beta_{1}}\left(\varphi_{p}\left(D_{0+}^{\alpha_{1}} u(t)\right)\right)+f_{1}(t, u(t), v(t))=0, & a<t<b, \\ D_{0+}^{\beta_{2}}\left(\varphi_{p}\left(D_{0+}^{\alpha_{2}} v(t)\right)\right)+f_{2}(t, u(t), v(t))=0, & a<t<b, \\ \xi u(a)-\eta u^{\prime}(a)=0, \gamma u(b)+\delta u^{\prime}(b)=0, & D_{0+}^{\alpha_{1}} u(a)=0, \\ \xi v(a)-\eta v^{\prime}(a)=0, \gamma v(b)+\delta v^{\prime}(b)=0, & D_{0+}^{\alpha_{2}} v(a)=0 .\end{cases}
$$


When the nonlinearities $f_{i}$ satisfy some appropriate conditions, the author made use of the Avery-Henderson fixed point theorem and the six functionals' fixed point theorem to obtain some existence theorems of multiple positive solutions.

Inspired by the aforementioned results, in this work, we study the solvability for (1) and establish the existence results of multiple positive solutions via the six functional fixed point theorem under some bounded conditions for $g_{i}(i=1,2)$. Finally, we also provide an example to illustrate our main results.

\section{Preliminaries}

In this section, we only recall the definition of Caputo fractional derivative, for more details, see the book [26].

Definition 1. The fractional derivative of $f$ in the Caputo sense is defined as
${ }^{c} D_{t}^{\mu} f(t)=\frac{1}{\Gamma(n-\mu)} \int_{0}^{t}(t-s)^{n-\mu-1} f^{(n)}(s) \mathrm{d} s, \quad n-1<\mu<n$,

where $n=[\mu]+1, \quad[\mu]$ denotes the integer part of the number $\mu$.

Now, we calculate Green's functions associated with (1). Let

$$
\begin{cases}\varphi_{p}\left({ }^{c} D_{t}^{\mu_{1}} u(t)\right)=x(t), & 0<t<1, \\ \varphi_{p}\left({ }^{c} D_{t}^{\mu_{2}} v(t)\right)=y(t), & 0<t<1,\end{cases}
$$

and then by the boundary conditions in (1), we have

$$
\begin{cases}x(0)=0, & x(1)=b_{1}^{p-1} x\left(\xi_{1}\right), \\ y(0)=0, & y(1)=b_{2}^{p-1} x\left(\xi_{2}\right) .\end{cases}
$$

Consequently, substituting (8) and (9) into (1), we obtain

$$
\begin{cases}{ }^{c} D_{t}^{\nu_{1}} x(t)+g_{1}(t, u(t), v(t))=0, & 0<t<1, \\ { }^{c} D_{t}^{\nu_{2}} y(t)+g_{2}(t, u(t), v(t))=0, & 0<t<1, \\ x(0)=0, & x(1)=b_{1}^{p-1} x\left(\xi_{1}\right), \\ y(0)=0, & y(1)=b_{2}^{p-1} x\left(\xi_{2}\right) .\end{cases}
$$

We next translate (10) into an equivalent system of integral equations. By the similar arguments as in [2], we have the following result.

Lemma 1. Problem (10) is equivalent to the following system of Hammerstein-type integral equations:

$$
\left(\begin{array}{l}
x(t) \\
y(t)
\end{array}\right)=\left(\begin{array}{l}
\int_{0}^{1} H_{1}(t, s) g_{1}(s, u(s), v(s)) \mathrm{d} s \\
\int_{0}^{1} H_{2}(t, s) g_{2}(s, u(s), v(s)) \mathrm{d} s
\end{array}\right)
$$

Proof. We only need to consider the case $i=1$ (by the similar method, the case $i=2$ can be easily proved). Using Lemma 2.5 of [2], we have

$$
x(t)=c_{0}+c_{1} t-\int_{0}^{t} \frac{(t-s)^{\nu_{1}-1}}{\Gamma\left(\nu_{1}\right)} g_{1}(s, u(s), v(s)) \mathrm{d} s,
$$

where $c_{i} \in \mathbb{R}, i=0,1 . x(0)=0$ implies that $c_{0}=0$, and by $x(1)=b_{1}^{p-1} x\left(\xi_{1}\right)$, we have

where

$$
\begin{aligned}
& H_{i}(t, s)=\bar{H}_{i}(t, s)+\frac{b_{i}^{p-1} t}{1-b_{i}^{p-1} \xi_{i}} \bar{H}_{i}\left(\xi_{i}, s\right), \quad t, s \in[0,1], \\
& \bar{H}_{i}(t, s)=\frac{1}{\Gamma\left(v_{i}\right)} \begin{cases}t(1-s)^{v_{i}-1}-(t-s)^{v_{i}-1}, & 0 \leq s \leq t \leq 1, \\
t(1-s)^{v_{i}-1}, & 0 \leq t \leq s \leq 1 .\end{cases}
\end{aligned}
$$

$$
\begin{aligned}
& c_{1}-\int_{0}^{1} \frac{(1-s)^{\nu_{1}-1}}{\Gamma\left(v_{1}\right)} g_{1}(s, u(s), v(s)) \mathrm{d} s=b_{1}^{p-1} \xi_{1} c_{1}-b_{1}^{p-1} \int_{0}^{\xi_{1}} \frac{\left(\xi_{1}-s\right)^{\nu_{1}-1}}{\Gamma\left(v_{1}\right)} g_{1}(s, u(s), v(s)) \mathrm{d} s, \\
& c_{1}=\frac{1}{1-b_{1}^{p-1} \xi_{1}} \int_{0}^{1} \frac{(1-s)^{\nu_{1}-1}}{\Gamma\left(v_{1}\right)} g_{1}(s, u(s), v(s)) \mathrm{d} s-\frac{b_{1}^{p-1}}{1-b_{1}^{p-1} \xi_{1}} \int_{0}^{\xi_{1}} \frac{\left(\xi_{1}-s\right)^{\nu_{1}-1}}{\Gamma\left(v_{1}\right)} g_{1}(s, u(s), v(s)) \mathrm{d} s .
\end{aligned}
$$


Therefore,

$$
\begin{aligned}
& x(t)=\frac{1}{1-b_{1}^{p-1} \xi_{1}} \int_{0}^{1} \frac{t(1-s)^{\nu_{1}-1}}{\Gamma\left(\nu_{1}\right)} g_{1}(s, u(s), v(s)) \mathrm{d} s \\
& -\frac{b_{1}^{p-1} t}{1-b_{1}^{p-1} \xi_{1}} \int_{0}^{\xi_{1}} \frac{\left(\xi_{1}-s\right)^{\nu_{1}-1}}{\Gamma\left(\nu_{1}\right)} g_{1}(s, u(s), v(s)) \mathrm{d} s-\int_{0}^{t} \frac{(t-s)^{\nu_{1}-1}}{\Gamma\left(\nu_{1}\right)} g_{1}(s, u(s), v(s)) \mathrm{d} s \\
& =\frac{1}{1-b_{1}^{p-1} \xi_{1}} \int_{0}^{1} \frac{t(1-s)^{\nu_{1}-1}}{\Gamma\left(\nu_{1}\right)} g_{1}(s, u(s), v(s)) \mathrm{d} s-\frac{b_{1}^{p-1} t}{1-b_{1}^{p-1} \xi_{1}} \int_{0}^{\xi_{1}} \frac{\left(\xi_{1}-s\right)^{\nu_{1}-1}}{\Gamma\left(\nu_{1}\right)} g_{1}(s, u(s), v(s)) \mathrm{d} s \\
& -\int_{0}^{1} \frac{t(1-s)^{\nu_{1}-1}}{\Gamma\left(\nu_{1}\right)} g_{1}(s, u(s), v(s)) \mathrm{d} s+\int_{0}^{1} \frac{t(1-s)^{\nu_{1}-1}}{\Gamma\left(\nu_{1}\right)} g_{1}(s, u(s), v(s)) \mathrm{d} s \\
& -\int_{0}^{t} \frac{(t-s)^{\nu_{1}-1}}{\Gamma\left(\nu_{1}\right)} g_{1}(s, u(s), v(s)) \mathrm{d} s=\int_{0}^{1} \bar{H}_{1}(t, s) g_{1}(s, u(s), v(s)) \mathrm{d} s \\
& +\frac{b_{1}^{p-1} \xi_{1}}{1-b_{1}^{p-1} \xi_{1}} \int_{0}^{1} \frac{t(1-s)^{\nu_{1}-1}}{\Gamma\left(\nu_{1}\right)} g_{1}(s, u(s), v(s)) \mathrm{d} s-\frac{b_{1}^{p-1} t}{1-b_{1}^{p-1} \xi_{1}} \int_{0}^{\xi_{1}} \frac{\left(\xi_{1}-s\right)^{\nu_{1}-1}}{\Gamma\left(\nu_{1}\right)} g_{1}(s, u(s), v(s)) \mathrm{d} s \\
& =\int_{0}^{1} \bar{H}_{1}(t, s) g_{1}(s, u(s), v(s)) \mathrm{d} s+\frac{b_{1}^{p-1} t}{1-b_{1}^{p-1} \xi_{1}} \int_{0}^{1} \bar{H}_{1}\left(\xi_{1}, s\right) g_{1}(s, u(s), v(s)) \mathrm{d} s \\
& =\int_{0}^{1} H_{1}(t, s) g_{1}(s, u(s), v(s)) \mathrm{d} s .
\end{aligned}
$$

This completes the proof.

Note from (8) and Lemma 1, we have

$$
\begin{cases}\varphi_{p}\left({ }^{c} D_{t}^{\mu_{1}} u(t)\right)=x(t)=\int_{0}^{1} H_{1}(t, s) g_{1}(s, u(s), v(s)) \mathrm{d} s, & 0<t<1, \\ \varphi_{p}\left({ }^{c} D_{t}^{\mu_{2}} v(t)\right)=y(t)=\int_{0}^{1} H_{2}(t, s) g_{2}(s, u(s), v(s)) \mathrm{d} s, & 0<t<1,\end{cases}
$$

and

$$
\begin{cases}\alpha_{1} u(0)-\beta_{1} u(1)=0, & \gamma_{1} u^{\prime}(0)-\delta_{1} u^{\prime}(1)=0, \\ \alpha_{2} v(0)-\beta_{2} v(1)=0, & \gamma_{2} v^{\prime}(0)-\delta_{2} v^{\prime}(1)=0 .\end{cases}
$$

$$
\begin{cases}{ }^{c} D_{t}^{\mu_{1}} u(t)=\varphi_{p}^{-1}\left(\int_{0}^{1} H_{1}(t, s) g_{1}(s, u(s), v(s)) \mathrm{d} s\right), & 0<t<1, \\ { }^{c} D_{t}^{\mu_{2}} v(t)=\varphi_{p}^{-1}\left(\int_{0}^{1} H_{2}(t, s) g_{2}(s, u(s), v(s)) \mathrm{d} s\right), & 0<t<1,\end{cases}
$$

By Lemma 2.5 of [2], we have the result:

Lemma 2. Problems (17) and (18) is equivalent to the following Hammerstein-type integral equation:

with the boundary conditions

$$
\left(\begin{array}{l}
u(t) \\
v(t)
\end{array}\right)=\left[\begin{array}{l}
\int_{0}^{1} G_{1}(t, s) \varphi_{p}^{-1}\left(\int_{0}^{1} H_{1}(s, \tau) g_{1}(\tau, u(\tau), v(\tau)) \mathrm{d} \tau\right) \mathrm{d} s \\
\int_{0}^{1} G_{2}(t, s) \varphi_{p}^{-1}\left(\int_{0}^{1} H_{2}(s, \tau) g_{2}(\tau, u(\tau), v(\tau)) \mathrm{d} \tau\right) \mathrm{d} s
\end{array}\right]
$$


where

$$
G_{i}(t, s)= \begin{cases}\frac{(t-s)^{\mu_{i}-1}}{\Gamma\left(\mu_{i}\right)}+\frac{\beta_{i}(1-s)^{\mu_{i}-1}}{\left(\alpha_{i}-\beta_{i}\right) \Gamma\left(\mu_{i}\right)}+\frac{\beta_{i} \delta_{i}\left(\mu_{i}-1\right)(1-s)^{\mu_{i}-2}}{\left(\alpha_{i}-\beta_{i}\right)\left(\gamma_{i}-\delta_{i}\right) \Gamma\left(\mu_{i}\right)}+\frac{\delta_{i}\left(\mu_{i}-1\right) t(1-s)^{\mu_{i}-2}}{\left(\gamma_{i}-\delta_{i}\right) \Gamma\left(\mu_{i}\right)}, & 0 \leq s \leq t \leq 1, \\ \frac{\beta_{i}(1-s)^{\mu_{i}-1}}{\left(\alpha_{i}-\beta_{i}\right) \Gamma\left(\mu_{i}\right)}+\frac{\beta_{i} \delta_{i}\left(\mu_{i}-1\right)(1-s)^{\mu_{i}-2}}{\left(\alpha_{i}-\beta_{i}\right)\left(\gamma_{i}-\delta_{i}\right) \Gamma\left(\mu_{i}\right)}+\frac{\delta_{i}\left(\mu_{i}-1\right) t(1-s)^{\mu_{i}-2}}{\left(\gamma_{i}-\delta_{i}\right) \Gamma\left(\mu_{i}\right)}, & 0 \leq t \leq s \leq 1 .\end{cases}
$$

Lemma 3 (see [2], Lemma 2.8). The functions $G_{i}, H_{i}(i=$ $1,2)$ have the following properties:

(i) $G_{i}, H_{i} \in C\left([0,1] \times[0,1], \mathbb{R}^{+}\right)$

(ii) $\mathscr{M}_{i}(s) \leq G_{i}(t, s) \leq\left(\alpha_{i} / \beta_{i}\right) \mathscr{M}_{i}(s), \forall t, s \in[0,1]$, where $\mathscr{M}_{i}(s)=\left(\beta_{i}(1-s)^{\mu_{i}-1} /\left(\alpha_{i}-\beta_{i}\right) \Gamma\left(\mu_{i}\right)\right)+\left(\beta_{i} \delta_{i}\left(\mu_{i}-\right.\right.$ 1) $\left.(1-s)^{\mu_{i}-2} /\left(\alpha_{i}-\beta_{i}\right)\left(\gamma_{i}-\delta_{i}\right) \Gamma\left(\mu_{i}\right)\right)$
Let $E:=C[0,1],\|u\|:=\max _{t \in[0,1]}|u(t)|$ and $P:=\{u \in E$ : $u(t) \geq 0, \forall t \in[0,1]\}$. Then, $(E,\|\cdot\|)$ is a real Banach space and $P$ is a cone on $E$. Moreover, $E \times E$ is a Banach space with the norm $\|(u, v)\|=\|u\|+\|v\|$, and $P \times P$ a cone on $E \times E$. From Lemmas 1 and 2, we define operators $T_{i}(i=1,2)$ and $T$ as follows:

$$
\begin{aligned}
& T_{1}(u, v)(t)=\int_{0}^{1} G_{1}(t, s) \varphi_{p}^{-1}\left(\int_{0}^{1} H_{1}(s, \tau) g_{1}(\tau, u(\tau), v(\tau)) \mathrm{d} \tau\right) \mathrm{d} s, \\
& T_{2}(u, v)(t)=\int_{0}^{1} G_{2}(t, s) \varphi_{p}^{-1}\left(\int_{0}^{1} H_{2}(s, \tau) g_{2}(\tau, u(\tau), v(\tau)) \mathrm{d} \tau\right) \mathrm{d} s, \\
& T(u, v)(t)=\left(T_{1}, T_{2}\right)(u, v)(t), \quad \text { for } t \in[0,1] .
\end{aligned}
$$

Then, we obtain that $T_{i}(i=1,2): P \times P \longrightarrow P, T: P \times$ $P \longrightarrow P \times P$ are completely continuous operators, and if there exists $(\bar{u}, \bar{v}) \in P \times P \backslash\{\mathbf{0}\}$ such that $T(\bar{u}, \bar{v})=(\bar{u}, \bar{v})$, i.e., $T_{1}(\bar{u}, \bar{v})=\bar{u}, T_{2}(\bar{u}, \bar{v})=\bar{v}$, then $(\bar{u}, \bar{v})$ is a positive solution for (1).

Lemma 4 (see [27]). Let $P$ be a cone in a real Banach space E. Suppose that $\alpha, \psi$, and $\zeta$ are nonnegative continuous concave functionals on $P, \beta, \sigma$, and $\theta$ are nonnegative continuous convex functionals on $P$, and there are nonnegative constants $l, l^{\prime}, r, r^{\prime}, R$, and $R^{\prime}$ such that $A: Q(\beta, R) \longrightarrow P$ is a completely continuous operator and

(B1) $Q(\beta, R)$ is a bounded set

(B2) $Q(\sigma, l)$ and $Q(\alpha, \beta, r, R)$ are disjoint subset of $Q(\beta, R)$

(B3) $\left\{u \in P\right.$ : $\left.\theta(u)<r^{\prime}, r<\alpha(u), R^{\prime}<\psi(u), \beta(u)<R\right\} \neq \varnothing$

(B4) $\left\{u \in P: l^{\prime}<\zeta(u), \sigma(u)<l\right\} \neq \varnothing$

(B5) $\{u \in P: l<\sigma(u), \alpha(u)<r\} \neq \varnothing$

If the following claims hold:

(C1) $\alpha(A u)>r, \forall u \in P$ with $\alpha(u)=r, \beta(u) \leq R$, and $r^{\prime}<\theta(A u)$

(C2) $\alpha(A u)>r, \forall u \in P$ with $\alpha(u)=r, \beta(u) \leq R$, and $\theta(u) \leq r^{\prime}$

(C3) $\beta(A u)<R, \forall u \in P$ with $r \leq \alpha(u), \beta(u)=R$, and $\psi(A u)<R^{\prime}$

(C4) $\beta(A u)<R, \forall u \in P$ with $r \leq \alpha(u), \beta(u)=R$, and $R^{\prime} \leq \psi(u)$

(C5) $\sigma(A u)<l, \forall u \in P$ with $\sigma(u)=l$ and $\zeta(A u)<l^{\prime}$
(C6) $\sigma(A u)<l, \forall u \in P$ with $\sigma(u)=l$ and $l^{\prime} \leq \zeta(u)$

Then, $A$ has at least three fixed points $u_{1}, u_{2}$, and $u_{3}$ in $Q(\beta, R)$ such that $\sigma\left(u_{1}\right) \leq l, r \leq\left(u_{2}\right)$ with $\beta\left(u_{2}\right) \leq R$, and $l<\sigma\left(u_{3}\right)$ with $\alpha\left(u_{3}\right)<r$.

\section{Main Results}

We first define some notations:

$$
\begin{aligned}
Q(\beta, R) & =\{(u, v) \in P \times P: \beta(u, v) \leq R\}, \\
Q(\alpha, \beta, r, R) & =\{(u, v) \in P \times P: r \leq \alpha(u, v) \text { and } \beta(u, v) \leq R\}, \\
\alpha(u, v) & =\min _{t \in[0,1]}\{u(t)+v(t)\}, \\
\psi(u, v) & =\min _{t \in I}\{u(t)+v(t)\}, \\
\zeta(u, v) & =\min _{t \in I_{1}}\{u(t)+v(t)\}, \\
\theta(u, v) & =\max _{t \in[0,1]}\{u(t)+v(t)\}, \\
\beta(u, v) & =\max _{t \in I}\{u(t)+v(t)\}, \\
\sigma(u, v) & =\max _{t \in I_{1}}\{u(t)+v(t)\},
\end{aligned}
$$

where $I=[(1 / 4),(3 / 4)]$ and $I_{1}=[(1 / 8),(7 / 8)]$. Then, $\alpha, \psi$, and $\zeta$ are the concave functionals on $P$, and $\theta, \beta$, and $\sigma$ are the convex functionals on $P$. 
Theorem 1. Suppose that there exist positive real numbers $l, l^{\prime}, r, r^{\prime}, R$, and $R^{\prime}$ such that $g_{i}(i=1,2)$ satisfy the following conditions:

(H2) $g_{1}(t, u(t), v(t))<\varphi_{p}\left(l \beta_{1} / 2 \alpha_{1} \rho_{1}\right), g_{2}(t, u(t), v(t))<$ $\varphi_{p}\left(l \beta_{2} / 2 \alpha_{2} \rho_{2}\right)$ for all $t \in[0,1]$ and $(u, v) \in[0,(r / 3)]$.

(H3) $\quad g_{1}(t, u(t), v(t))<\varphi_{p}\left(R \beta_{1} / 2 \alpha_{1} \rho_{1}\right), \quad g_{2}(t, u(t)$, $v(t))<\varphi_{p}\left(R \beta_{2} / 2 \alpha_{2} \rho_{2}\right)$ for all $t \in[0,1]$ and $(u, v) \in[0, R]$. (H4) $g_{1}(t, u(t), v(t))<\varphi_{p}\left(r / 2 \rho_{1}\right), g_{2}(t, u(t), v(t))<$ $\varphi_{p}\left(r / 2 \rho_{2}\right)$ for all $t \in[0,1]$ and $(u, v) \in[r,(r / \eta)]$, where $\eta=\min \left\{\left(\beta_{1} / \alpha_{1}\right),\left(\beta_{2} / \alpha_{2}\right)\right\}$ :

$$
\begin{aligned}
& \rho_{1}=\int_{0}^{1} \mathscr{M}_{1}(s) \varphi_{p}^{-1}\left(\int_{0}^{1} H_{1}(s, \tau) \mathrm{d} \tau\right) \mathrm{d} s, \\
& \rho_{2}=\int_{0}^{1} \mathscr{M}_{2}(s) \varphi_{p}^{-1}\left(\int_{0}^{1} H_{2}(s, \tau) \mathrm{d} \tau\right) \mathrm{d} s .
\end{aligned}
$$

Then, (1) has at least three positive solutions $\left(u_{1}, v_{1}\right)$, $\left(u_{2}, v_{2}\right)$, and $\left(u_{3}, v_{3}\right)$ in $Q(\beta, R)$.

Proof. Note, from a standard calculus argument, we obtain the set $Q(\beta, R)$ is bounded. Since if $(u, v) \in Q(\beta, R)$, and then $\max _{t \in I}\{u(t)+v(t)\}<R$. Let $r^{\prime}=(r / \eta), R=\left(R^{\prime} / \eta\right)$, $l=\left(l^{\prime} / \eta\right)$, and $l=(r / 3)$ with $R^{\prime} \in\left(\left(2 \eta^{2} r^{\prime} /(\eta+1)\right)\right.$, $\left.\left(2 \eta r^{\prime} /(\eta+1)\right)\right)$. Then, if $(u, v) \in Q(\sigma, l)$, we have $\max _{t \in I_{1}}\{u(t)+v(t)\}<l$. This means that

$$
\begin{aligned}
\alpha(u, v) & =\min _{t \in[0,1]}\{u(t)+v(t)\} \leq \min _{t \in I_{1}}\{u(t)+v(t)\} \\
& \leq \max _{t \in I_{1}}\{u(t)+v(t)\}<l<r,
\end{aligned}
$$

and $Q(\sigma, l) \cap Q(\alpha, \beta, r, R)=\varnothing$. Also, it can easily be shown that

$$
\begin{aligned}
&\left(\frac{R^{\prime}}{2}, \frac{R}{2}\right) \in\left\{(u, v) \in P: \theta(u, v)<r^{\prime}, r<\alpha(u, v), R^{\prime}\right. \\
&<\psi(u, v) \text { and } \beta(u, v)<R\}, \\
&\left(\frac{\eta l}{2}, \frac{l}{2}\right) \in\left\{(u, v) \in P: l^{\prime}<\zeta(u, v) \text { and } \sigma(u, v)<l\right\}, \\
&\left(\frac{r}{2}, \frac{l}{2}\right) \in\{(u, v) \in P: l<\sigma(u, v) \text { and } \alpha(u, v)<r\} .
\end{aligned}
$$

Hence, these sets are nonempty. As a result, (B1)-(B5) of Lemma 4 are satisfied.

Now, we verify the functional conditions.

Claim 1. $\alpha(T(u, v))>r$, for all $(u, v) \in Q(\alpha, \beta, r, R)$ with $\alpha(u, v)=r$ and $r^{\prime}<\theta(T(u, v))$. Note, from Lemma 3 (ii), we have

$$
\begin{aligned}
\alpha(T(u, v)) & =\min _{t \in[0,1]}\left\{T_{1}(u, v)(t)+T_{2}(u, v)(t)\right\} \\
& =\min _{t \in[0,1]}\left\{\int_{0}^{1} G_{1}(t, s) \varphi_{p}^{-1}\left(\int_{0}^{1} H_{1}(s, \tau) g_{1}(\tau, u(\tau), v(\tau)) \mathrm{d} \tau\right) \mathrm{d} s+\int_{0}^{1} G_{2}(t, s) \varphi_{p}^{-1}\left(\int_{0}^{1} H_{2}(s, \tau) g_{2}(\tau, u(\tau), v(\tau)) \mathrm{d} \tau\right) \mathrm{d} s\right\} \\
& \geq \int_{0}^{1} \mathscr{M}_{1}(s) \varphi_{p}^{-1}\left(\int_{0}^{1} H_{1}(s, \tau) g_{1}(\tau, u(\tau), v(\tau)) \mathrm{d} \tau\right) \mathrm{d} s+\int_{0}^{1} \mathscr{M}_{2}(s) \varphi_{p}^{-1}\left(\int_{0}^{1} H_{2}(s, \tau) g_{2}(\tau, u(\tau), v(\tau)) \mathrm{d} \tau\right) \mathrm{d} s \\
& =\frac{\beta_{1}}{\alpha_{1}} \int_{0}^{1} \frac{\alpha_{1}}{\beta_{1}} \mathscr{M}_{1}(s) \varphi_{p}^{-1}\left(\int_{0}^{1} H_{1}(s, \tau) g_{1}(\tau, u(\tau), v(\tau)) \mathrm{d} \tau\right) \mathrm{d} s+\frac{\beta_{2}}{\alpha_{2}} \int_{0}^{1} \frac{\alpha_{2}}{\beta_{2}} \mathscr{M}_{2}(s) \varphi_{p}^{-1}\left(\int_{0}^{1} H_{2}(s, \tau) g_{2}(\tau, u(\tau), v(\tau)) \mathrm{d} \tau\right) \mathrm{d} s \\
& \geq \eta \max _{t \in[0,1]}\left\{\int_{0}^{1} G_{1}(t, s) \varphi_{p}^{-1}\left(\int_{0}^{1} H_{1}(s, \tau) g_{1}(\tau, u(\tau), v(\tau)) \mathrm{d} \tau\right) \mathrm{d} s+\int_{0}^{1} G_{2}(t, s) \varphi_{p}^{-1}\left(\int_{0}^{1} H_{2}(s, \tau) g_{2}(\tau, u(\tau), v(\tau)) \mathrm{d} \tau\right) \mathrm{d} s\right\} \\
& =\eta \theta(T(u, v)) \\
& >\eta r^{\prime} \\
& =r .
\end{aligned}
$$

Claim 2. $\alpha(T(u, v))>r$, for all $(u, v) \in\{(u, v) \in$ $\left.Q(\alpha, \beta, r, R): \theta(u, v) \leq r^{\prime}\right\}$ with $\alpha(u, v)=r$. At this case, we have $r \leq u(\tau)+v(\tau) \leq(r / \eta)$, for $\tau \in[0,1]$, and from (H4), we obtain 


$$
\begin{aligned}
\alpha(T(u, v)) & =\min _{t \in[0,1]}\left\{T_{1}(u, v)(t)+T_{2}(u, v)(t)\right\} \\
& =\min _{t \in[0,1]}\left\{\int_{0}^{1} G_{1}(t, s) \varphi_{p}^{-1}\left(\int_{0}^{1} H_{1}(s, \tau) g_{1}(\tau, u(\tau), v(\tau)) \mathrm{d} \tau\right) \mathrm{d} s+\int_{0}^{1} G_{2}(t, s) \varphi_{p}^{-1}\left(\int_{0}^{1} H_{2}(s, \tau) g_{2}(\tau, u(\tau), v(\tau)) \mathrm{d} \tau\right) \mathrm{d} s\right\} \\
& \geq \int_{0}^{1} \mathscr{M}_{1}(s) \varphi_{p}^{-1}\left(\int_{0}^{1} H_{1}(s, \tau) g_{1}(\tau, u(\tau), v(\tau)) \mathrm{d} \tau\right) \mathrm{d} s+\int_{0}^{1} \mathscr{M}_{2}(s) \varphi_{p}^{-1}\left(\int_{0}^{1} H_{2}(s, \tau) g_{2}(\tau, u(\tau), v(\tau)) \mathrm{d} \tau\right) \mathrm{d} s \\
& >\int_{0}^{1} \mathscr{M}_{1}(s) \varphi_{p}^{-1}\left(\int_{0}^{1} H_{1}(s, \tau) \varphi_{p}\left(\frac{r}{2 \rho_{1}}\right) \mathrm{d} \tau\right) \mathrm{d} s+\int_{0}^{1} \mathscr{M}_{2}(s) \varphi_{p}^{-1}\left(\int_{0}^{1} H_{2}(s, \tau) \varphi_{p}\left(\frac{r}{2 \rho_{2}}\right) \mathrm{d} \tau\right) \mathrm{d} s \\
& =\frac{r}{2 \rho_{1}} \int_{0}^{1} \mathscr{M}_{1}(s) \varphi_{p}^{-1}\left(\int_{0}^{1} H_{1}(s, \tau) \mathrm{d} \tau\right) \mathrm{d} s+\frac{r}{2 \rho_{2}} \int_{0}^{1} \mathscr{M}_{2}(s) \varphi_{p}^{-1}\left(\int_{0}^{1} H_{2}(s, \tau) \mathrm{d} \tau\right) \mathrm{d} s \\
& =\frac{r}{2}+\frac{r}{2} \\
& =r .
\end{aligned}
$$

Claim 3. $\beta(T(u, v))<R$, for all $(u, v) \in Q(\alpha, \beta, r, R)$,

$\beta(u, v)=R$, and $\psi(T(u, v))<R^{\prime}$. Then, we have

$$
\begin{aligned}
\beta(T(u, v)) & =\max _{t \in I}\left\{T_{1}(u, v)(t)+T_{2}(u, v)(t)\right\} \\
& =\max _{t \in I}\left\{\int_{0}^{1} G_{1}(t, s) \varphi_{p}^{-1}\left(\int_{0}^{1} H_{1}(s, \tau) g_{1}(\tau, u(\tau), v(\tau)) \mathrm{d} \tau\right) \mathrm{d} s+\int_{0}^{1} G_{2}(t, s) \varphi_{p}^{-1}\left(\int_{0}^{1} H_{2}(s, \tau) g_{2}(\tau, u(\tau), v(\tau)) \mathrm{d} \tau\right) \mathrm{d} s\right\} \\
& \leq \int_{0}^{1} \frac{\alpha_{1}}{\beta_{1}} \mathscr{M}_{1}(s) \varphi_{p}^{-1}\left(\int_{0}^{1} H_{1}(s, \tau) g_{1}(\tau, u(\tau), v(\tau)) \mathrm{d} \tau\right) \mathrm{d} s+\int_{0}^{1} \frac{\alpha_{2}}{\beta_{2}} \mathscr{M}_{2}(s) \varphi_{p}^{-1}\left(\int_{0}^{1} H_{2}(s, \tau) g_{2}(\tau, u(\tau), v(\tau)) \mathrm{d} \tau\right) \mathrm{d} s \\
& \leq \frac{1}{\eta}\left\{\int_{0}^{1} \mathscr{M}_{1}(s) \varphi_{p}^{-1}\left(\int_{0}^{1} H_{1}(s, \tau) g_{1}(\tau, u(\tau), v(\tau)) \mathrm{d} \tau\right) \mathrm{d} s+\int_{0}^{1} \mathscr{M}_{2}(s) \varphi_{p}^{-1}\left(\int_{0}^{1} H_{2}(s, \tau) g_{2}(\tau, u(\tau), v(\tau)) \mathrm{d} \tau\right) \mathrm{d} s\right\} \\
& \leq \frac{1}{\eta} \min _{t \in I}\left\{\int_{0}^{1} G_{1}(t, s) \varphi_{p}^{-1}\left(\int_{0}^{1} H_{1}(s, \tau) g_{1}(\tau, u(\tau), v(\tau)) \mathrm{d} \tau\right) \mathrm{d} s+\int_{0}^{1} G_{2}(t, s) \varphi_{p}^{-1}\left(\int_{0}^{1} H_{2}(s, \tau) g_{2}(\tau, u(\tau), v(\tau)) \mathrm{d} \tau\right) \mathrm{d} s\right\} \\
& =\frac{1}{\eta} \psi(u, v) \\
& <\frac{R^{\prime}}{\eta} \\
& =R .
\end{aligned}
$$


Claim 4. $\beta(T(u, v))<R$, for all $(u, v) \in\{(u, v) \in$ $\left.Q(\alpha, \beta, r, R): R^{\prime} \leq \psi(u, v)\right\}$ with $\beta(u, v)=R$. Therefore, by Lemma 3 (ii) and (H3), we have

$$
\begin{aligned}
\beta(T(u, v)) & =\max _{t \in I}\left\{T_{1}(u, v)(t)+T_{2}(u, v)(t)\right\} \\
& =\max _{t \in I}\left\{\int_{0}^{1} G_{1}(t, s) \varphi_{p}^{-1}\left(\int_{0}^{1} H_{1}(s, \tau) g_{1}(\tau, u(\tau), v(\tau)) \mathrm{d} \tau\right) \mathrm{d} s+\int_{0}^{1} G_{2}(t, s) \varphi_{p}^{-1}\left(\int_{0}^{1} H_{2}(s, \tau) g_{2}(\tau, u(\tau), v(\tau)) \mathrm{d} \tau\right) \mathrm{d} s\right\} \\
& \leq \int_{0}^{1} \frac{\alpha_{1}}{\beta_{1}} \mathscr{M}_{1}(s) \varphi_{p}^{-1}\left(\int_{0}^{1} H_{1}(s, \tau) g_{1}(\tau, u(\tau), v(\tau)) \mathrm{d} \tau\right) \mathrm{d} s+\int_{0}^{1} \frac{\alpha_{2}}{\beta_{2}} \mathscr{M}_{2}(s) \varphi_{p}^{-1}\left(\int_{0}^{1} H_{2}(s, \tau) g_{2}(\tau, u(\tau), v(\tau)) \mathrm{d} \tau\right) \mathrm{d} s \\
& <\int_{0}^{1} \frac{\alpha_{1}}{\beta_{1}} \mathscr{M}_{1}(s) \varphi_{p}^{-1}\left(\int_{0}^{1} H_{1}(s, \tau) \varphi_{p}\left(\frac{R \beta_{1}}{2 \alpha_{1} \rho_{1}}\right) \mathrm{d} \tau\right) \mathrm{d} s+\int_{0}^{1} \frac{\alpha_{2}}{\beta_{2}} \mathscr{M}_{2}(s) \varphi_{p}^{-1}\left(\int_{0}^{1} H_{2}(s, \tau) \varphi_{p}\left(\frac{R \beta_{2}}{2 \alpha_{2} \rho_{2}}\right) \mathrm{d} \tau\right) \mathrm{d} s \\
& =\frac{R \beta_{1}}{2 \alpha_{1} \rho_{1}} \int_{0}^{1} \frac{\alpha_{1}}{\beta_{1}} \mathscr{M}_{1}(s) \varphi_{p}^{-1}\left(\int_{0}^{1} H_{1}(s, \tau) \mathrm{d} \tau\right) \mathrm{d} s+\frac{R \beta_{2}}{2 \alpha_{2} \rho_{2}} \int_{0}^{1} \frac{\alpha_{2}}{\beta_{2}} \mathscr{M}_{2}(s) \varphi_{p}^{-1}\left(\int_{0}^{1} H_{2}(s, \tau) \mathrm{d} \tau\right) \mathrm{d} s \\
& =\frac{R}{2}+\frac{R}{2} \\
& =R .
\end{aligned}
$$

Claim 5. $\sigma(T(u, v))<l$, for all $(u, v) \in Q(\sigma, l)$ with $\sigma(u, v)=l$, and $\zeta(T(u, v))<l^{\prime}$. Then, we obtain

$$
\begin{aligned}
\sigma(T(u, v)) & =\max _{t \in I_{1}}\left\{T_{1}(u, v)(t)+T_{2}(u, v)(t)\right\} \\
& =\max _{t \in I_{1}}\left\{\int_{0}^{1} G_{1}(t, s) \varphi_{p}^{-1}\left(\int_{0}^{1} H_{1}(s, \tau) g_{1}(\tau, u(\tau), v(\tau)) \mathrm{d} \tau\right) \mathrm{d} s+\int_{0}^{1} G_{2}(t, s) \varphi_{p}^{-1}\left(\int_{0}^{1} H_{2}(s, \tau) g_{2}(\tau, u(\tau), v(\tau)) \mathrm{d} \tau\right) \mathrm{d} s\right\} \\
& \leq \int_{0}^{1} \frac{\alpha_{1}}{\beta_{1}} \mathscr{M}_{1}(s) \varphi_{p}^{-1}\left(\int_{0}^{1} H_{1}(s, \tau) g_{1}(\tau, u(\tau), v(\tau)) \mathrm{d} \tau\right) \mathrm{d} s+\int_{0}^{1} \frac{\alpha_{2}}{\beta_{2}} \mathscr{M}_{2}(s) \varphi_{p}^{-1}\left(\int_{0}^{1} H_{2}(s, \tau) g_{2}(\tau, u(\tau), v(\tau)) \mathrm{d} \tau\right) \mathrm{d} s \\
& \leq \frac{1}{\eta}\left\{\int_{0}^{1} \mathscr{M}_{1}(s) \varphi_{p}^{-1}\left(\int_{0}^{1} H_{1}(s, \tau) g_{1}(\tau, u(\tau), v(\tau)) \mathrm{d} \tau\right) \mathrm{d} s+\int_{0}^{1} \mathscr{M}_{2}(s) \varphi_{p}^{-1}\left(\int_{0}^{1} H_{2}(s, \tau) g_{2}(\tau, u(\tau), v(\tau)) \mathrm{d} \tau\right) \mathrm{d} s\right\} \\
& \leq \frac{1}{\eta} \min _{t \in I_{1}}\left\{\int_{0}^{1} G_{1}(t, s) \varphi_{p}^{-1}\left(\int_{0}^{1} H_{1}(s, \tau) g_{1}(\tau, u(\tau), v(\tau)) \mathrm{d} \tau\right) \mathrm{d} s+\int_{0}^{1} G_{2}(t, s) \varphi_{p}^{-1}\left(\int_{0}^{1} H_{2}(s, \tau) g_{2}(\tau, u(\tau), v(\tau)) \mathrm{d} \tau\right) \mathrm{d} s\right\} \\
& =\frac{1}{\eta} \zeta(T(u, v)) \\
& <\frac{l^{\prime}}{\eta} \\
& =l .
\end{aligned}
$$


Claim 6. $\sigma(T(u, v))<l$, for all $(u, v) \in\left\{Q(\sigma, l): l^{\prime} \leq \zeta(u, v)\right\}$ with $\sigma(T(u, v))<l$. Therefore, by Lemma 3 (ii) and (H2), we have

$$
\begin{aligned}
\sigma(T(u, v)) & =\max _{t \in I_{1}}\left\{T_{1}(u, v)(t)+T_{2}(u, v)(t)\right\} \\
& =\max _{t \in I_{1}}\left\{\int_{0}^{1} G_{1}(t, s) \varphi_{p}^{-1}\left(\int_{0}^{1} H_{1}(s, \tau) g_{1}(\tau, u(\tau), v(\tau)) \mathrm{d} \tau\right) \mathrm{d} s+\int_{0}^{1} G_{2}(t, s) \varphi_{p}^{-1}\left(\int_{0}^{1} H_{2}(s, \tau) g_{2}(\tau, u(\tau), v(\tau)) \mathrm{d} \tau\right) \mathrm{d} s\right\} \\
& \leq \int_{0}^{1} \frac{\alpha_{1}}{\beta_{1}} \mathscr{M}_{1}(s) \varphi_{p}^{-1}\left(\int_{0}^{1} H_{1}(s, \tau) g_{1}(\tau, u(\tau), v(\tau)) \mathrm{d} \tau\right) \mathrm{d} s+\int_{0}^{1} \frac{\alpha_{2}}{\beta_{2}} \mathscr{M}_{2}(s) \varphi_{p}^{-1}\left(\int_{0}^{1} H_{2}(s, \tau) g_{2}(\tau, u(\tau), v(\tau)) \mathrm{d} \tau\right) \mathrm{d} s \\
& <\int_{0}^{1} \frac{\alpha_{1}}{\beta_{1}} M_{1}(s) \varphi_{p}^{-1}\left(\int_{0}^{1} H_{1}(s, \tau) \varphi_{p}\left(\frac{l \beta_{1}}{2 \alpha_{1} \rho_{1}}\right) \mathrm{d} \tau\right) \mathrm{d} s+\int_{0}^{1} \frac{\alpha_{2}}{\beta_{2}} M_{2}(s) \varphi_{p}^{-1}\left(\int_{0}^{1} H_{2}(s, \tau) \varphi_{p}\left(\frac{l \beta_{2}}{2 \alpha_{2} \rho_{2}}\right) \mathrm{d} \tau\right) \mathrm{d} s \\
& =\frac{l \beta_{1}}{2 \alpha_{1} \rho_{1}} \int_{0}^{1} \frac{\alpha_{1}}{\beta_{1}} M_{1}(s) \varphi_{p}^{-1}\left(\int_{0}^{1} H_{1}(s, \tau) \mathrm{d} \tau\right) \mathrm{d} s+\frac{l \beta_{2}}{2 \alpha_{2} \rho_{2}} \int_{0}^{1} \frac{\alpha_{2}}{\beta_{2}} M_{2}(s) \varphi_{p}^{-1}\left(\int_{0}^{1} H_{2}(s, \tau) \mathrm{d} \tau\right) \mathrm{d} s \\
& =\frac{l}{2}+\frac{l}{2} \\
& =l
\end{aligned}
$$

Remark 1. Our Green's functions $G_{i}(i=1,2)$ satisfy some special inequalities (see Lemma 3 (ii)). So, in the Claims 1 and 2 of Theorem 1, $\alpha(T(u, v))=\min _{t \in[0,1]}\left\{T_{1}(u, v)(t)+\right.$ $\left.T_{2}(u, v)(t)\right\}$ cannot reach zero. Therefore, we use the Caputo derivative for the considered scheme. This also explains the reason that we do not use other forms of fractional derivatives (including the Riemann-Liouville type and the Hadamard type).

Example 1. Consider the example: where $p=2$.

In order to obtain the constants in Theorem 1, we need to calculate

$$
\begin{aligned}
\int_{0}^{1} \bar{H}_{i}(t, s) \mathrm{d} s & =\frac{1}{\Gamma\left(v_{i}\right)} \int_{0}^{1} t(1-s)^{v_{i}-1} \mathrm{~d} s-\frac{1}{\Gamma\left(v_{i}\right)} \int_{0}^{t}(t-s)^{v_{i}-1} \mathrm{~d} s \\
& =\left.\frac{t}{v_{i} \Gamma\left(v_{i}\right)}\left[-(1-s)^{v_{i}}\right]\right|_{0} ^{1}+\left.\frac{1}{v_{i} \Gamma\left(v_{i}\right)}(t-s)^{v_{i}}\right|_{0} ^{t} \\
& =\frac{t-t^{v_{i}}}{\Gamma\left(v_{i}+1\right)}
\end{aligned}
$$

and we have 
Moreover, we also obtain that $\eta=0.5, b_{i}^{p-1} \xi_{i}=0.25$, $b_{1}^{p-1} \xi_{1}^{\nu_{1}}=0.125, b_{2}^{p-1} \xi_{2}^{\nu_{2}}=0.088, \quad \int_{0}^{1} H_{1}(t, s) \mathrm{d} s=0.878 t-$ $0.752 t^{1.5}, \quad \int_{0}^{1} H_{2}(t, s) \mathrm{d} s=0.915 t-0.752 t^{1.5}, \quad \mathscr{M}_{1}(s)=$ $1.13(1-s)^{0.5}+2.82(1-s)^{-0.5}, \quad \mathscr{M}_{2}(s)=3.39(1-s)^{0.5}+$ $11.85(1-s)^{-0.5}, \quad \rho_{1}=0.9$, and $\rho_{2}=4.29$. If we put $l=2, l^{\prime}=1, r=6, r^{\prime}=12, R=15.6, R^{\prime}=7.8$, and

$$
\begin{aligned}
& g_{1}(t, u, v)= \begin{cases}\frac{t}{60}+0.25(u+v), & 0 \leq u+v \leq 2, t \in[0,1], \\
\frac{t}{60}+0.875(u+v)-1.25, & 2 \leq u+v \leq 6, t \in[0,1], \\
\frac{t}{60}+4, & 6 \leq u+v \leq 12, t \in[0,1], \\
\frac{t}{60}-0.14(u+v)+5.68, & 12 \leq u+v \leq 15.6, t \in[0,1],\end{cases} \\
& g_{2}(t, u, v)= \begin{cases}\frac{t}{70}+0.08(u+v), & 0 \leq u+v \leq 2, t \in[0,1], \\
\frac{t}{70}+0.21(u+v)-0.26, & 2 \leq u+v \leq 6, t \in[0,1], \\
\frac{t}{70}+1, & 6 \leq u+v \leq 12, t \in[0,1], \\
\frac{t}{70}+0.056(u+v)+0.328, & 12 \leq u+v \leq 15.6, t \in[0,1] .\end{cases}
\end{aligned}
$$

Then, $g_{i}(i=1,2)$ satisfies the following conditions:

(i) $g_{1}(t, u(t), v(t))<0.56, g_{2}(t, u(t), v(t))<0.18$ for all $t \in[0,1]$ and $(u, v) \in[0,2]$

(ii) $g_{1}(t, u(t), v(t))<4.33, g_{2}(t, u(t), v(t))<1.37 \quad$ for all $t \in[0,1]$ and $(u, v) \in[0,15.6]$

(iii) $g_{1}(t, u(t), v(t))>3.33, g_{2}(t, u(t), v(t))>0.7$ for all $t \in[0,1]$ and $(u, v) \in[6,12]$

Therefore, all assumptions in Theorem 1 are satisfied, and the system of (32) has at least triple positive solutions.

\section{Conclusion}

In this paper, we use the six functional fixed point theorem to study the system of (1) under some bounded conditions for $g_{i}(i=1,2)$. We first transform the original fractional differential system into the equivalent system of Hammerstein-type integral equations, and then with the help of properties of Green's functions, we provide some sufficient conditions to guarantee the existence of multiple positive solutions for our system. Finally, an example is presented to illustrate the effectiveness of the main result.

\section{Data Availability}

No data were used to support this study.

\section{Conflicts of Interest}

The authors declare that there are no conflicts of interest regarding the publication of this paper.

\section{Acknowledgments}

This work was supported by the National Natural Science Foundation of China (11771017), China Postdoctoral Science Foundation (2019M652348), Key Research of Henan Higher Education Institutions (17A11001, 19A110018, and 20B110006), Technology Research Foundation of Chongqing Educational Committee (KJQN201900539), Fundamental Research Funds for the Universities of Henan Province (NSFRF180320), and Henan Polytechnic University Doctor Fund (B2016-58).

\section{References}

[1] S. Vong, "Positive solutions of singular fractional diferential equations with integral boundary conditions," Mathematical and Computer Modelling, vol. 57, no. 5-6, pp. 1053-1059, 2013.

[2] Y. Wang and Y. Yang, "Positive solutions for Caputo fractional differential equations involving integral boundary conditions," Journal of Nonlinear Sciences and Applications, vol. 8, no. 2, pp. 99-109, 2015. 
[3] F. Wang, L. Liu, Y. Wu, and Y. Zou, "Iterative analysis of the unique positive solution for a class of singular nonlinear boundary value problems involving two types of fractional derivatives with $p$-Laplacian operator," Complexity, vol. 2019, Article ID 2319062, 21 pages, 2019.

[4] J. Xu, J. Jiang, and D. O'Regan, "Positive solutions for a class of $p$-Laplacian Hadamard fractional-order three-point boundary value problems," Mathematics, vol. 8, no. 3, p. 308, 2020.

[5] J. Jiang, D. O'Regan, J. Xu, and Y. Cui, “Positive solutions for a Hadamard fractional $p$-Laplacian three-point boundary value problem," Mathematics, vol. 7, no. 5, p. 439, 2019.

[6] W. Cheng, J. Xu, D. O’Regan, and Y. Cui, "Positive solutions for a nonlinear discrete fractional boundary value problem with a $p$-Laplacian operator," Journal of Applied Analysis and Computation, vol. 9, no. 5, pp. 1959-1972, 2019.

[7] Z. Han, H. Lu, and C. Zhang, "Positive solutions for eigenvalue problems of fractional diferential equation with generalized p-Laplacian," Applied Mathematics and Computation, vol. 257, pp. 526-536, 2015.

[8] S. K. Ntouyas and S. Etemad, "On the existence of solutions for fractional diferential inclusions with sum and integral boundary conditions," Applied Mathematics and Computation, vol. 266, pp. 235-243, 2015.

[9] Z. Fu, S. Bai, D. O’Regan, and J. Xu, "Nontrivial solutions for an integral boundary value problem involving RiemannLiouville fractional derivatives," Journal of Inequalities and Applications, vol. 2019, no. 1, 2019.

[10] X. Zhang and Y. Han, "Existence and uniqueness of positive solutions for higher order nonlocal fractional diferential equations," Applied Mathematics Letters, vol. 25, no. 3, pp. 555-560, 2012.

[11] Y. Wang, "Multiple positive solutions for mixed fractional differential system with $p$-Laplacian operators," Boundary Value Problems, vol. 2019, no. 1, 2019.

[12] S. N. Rao, "Multiplicity of positive solutions for coupled system of fractional differential equation with $p$-Laplacian two-point BVPs," Journal of Applied Mathematics and Computing, vol. 55, no. 1-2, pp. 41-58, 2017.

[13] B. M. B. Krushna, "Eigenvalues for iterative systems of Riemann-Liouville type $p$-Laplacian fractional-order boundaryvalue problems in Banach spaces," Computational and Applied Mathematics, vol. 39, no. 2, 2020.

[14] Y. Wang and J. Jiang, "Existence and nonexistence of positive solutions for the fractional coupled system involving generalized p-Laplacian," Advances in Difference Equations, vol. 2017, no. 1, 2017.

[15] S. Li, X. Zhang, Y. Wu, and L. Caccetta, "Extremal solutions for $p$-Laplacian differential systems via iterative computation," Applied Mathematics Letters, vol. 26, no. 12, pp. 1151-1158, 2013.

[16] J. Henderson, R. Luca, and A. Tudorache, "On a system of fractional diferential equations with coupled integral boundary conditions," Fractional Calculus and Applied Analysis, vol. 18, no. 2, pp. 361-386, 2015.

[17] C. Yuan, D. Jiang, D. O’Regan, and R. P. Agarwal, “Multiple positive solutions to systems of nonlinear semipositone fractional diferential equations with coupled boundary conditions," Electronic Journal of Qualitative Theory of Differential Equations, vol. 13, p. 17, 2012.

[18] N. A. Asif and R. A. Khan, "Positive solutions to singular system with four-point coupled boundary conditions," Journal of Mathematical Analysis and Applications, vol. 386, no. 2, pp. 848-861, 2012.
[19] J. Jiang, "Solvability of anti-periodic boundary value problem for coupled system of fractional $p$-Laplacian equation," Advances in Difference Equations, vol. 2015, no. 1, 2015.

[20] A. Y. Al-Hossain, "Existence of positive solutions for system of p-Laplacian fractional order boundary value problems," Differential Equations and Dynamical Systems, vol. 27, no. 4, pp. 539-552, 2019.

[21] Y. Ding, J. Jiang, D. O’Regan, and J. Xu, "Positive solutions for a system of Hadamard-type fractional differential equations with semipositone nonlinearities," Complexity, vol. 2020, Article ID 9742418, 14 pages, 2020.

[22] X. Hao, H. Wang, L. Liu, and Y. Cui, "Positive solutions for a system of nonlinear fractional nonlocal boundary value problems with parameters and p-Laplacian operator," Boundary Value Problems, vol. 2017, no. 1, 2017.

[23] J. Xu, C. S. Goodrich, and Y. Cui, "Positive solutions for a system of first-order discrete fractional boundary value problems with semipositone nonlinearities," Revista de la Real Academia de Ciencias Exactas, Físicas y Naturales. Serie A. Matemáticas, vol. 113, no. 2, pp. 1343-1358, 2019.

[24] W. Cheng, J. Xu, Y. Cui, and Q. Ge, "Positive solutions for a class of fractional difference systems with coupled boundary conditions," Advances in Difference Equations, vol. 2019, no. 1, 2019.

[25] H. Zhang, Y. Li, and J. Xu, "Positive solutions for a system of fractional integral boundary value problems involving Hadamard-type fractional derivatives," Complexity, vol. 2019, Article ID 2671539, 11 pages, 2019.

[26] A. A. Kilbas, H. M. Srivastava, and J. J. Trujillo, Theory and Applications of Fractional Differential Equations, Elsevier, Boston, MA, USA, 2006.

[27] R. Avery, J. Henderson, and D. O’Regan, "Six functionals fixed point theorem," Journal of Computational Analysis and Applications, vol. 12, pp. 69-82, 2008. 